\title{
Measurement of W-band gyro-TWA with wide bandwidth input signal
}

\author{
Liang Zhang, Craig R. Donaldson, Adrian W. Cross, \\ Wenlong He \\ Department of Physics, SUPA, \\ University of Strathclyde \\ Glasgow, UK
}

\author{
Peter Cain \\ Keysight Technologies UK Ltd, \\ St. Andrews, UK
}

\begin{abstract}
A W-band gyro-TWA has been developed to provide high output power and wide bandwidth. This letter presents the upgrade of the input signal generation of the $\mathrm{W}$-band gyro-TWA, and the experiment results with frequency-swept input signals. The results show a $30 \mathrm{~dB}$ gain was achieved at a 1 GHz bandwidth input signal when the gyro-TWA was driven by a $40 \mathrm{kV}, 1.5$ A electron beam.
\end{abstract}

Keywords-gyrotron traveling wave amplifier, helically corrugated waveguide, frequency-swept

\section{INTRODUCTION}

Gyrotron traveling wave amplifier (gyro-TWA) is one of the gyro-devices that is able to produce high power coherent microwave radiation at mm-wave and sub-mm-wave frequencies. Its amplitude and phase can be precisely controlled therefore it is attractive in applications such as communications, radar and electron spin resonance spectroscopy.

A helically corrugated waveguide can be as the interaction region of the gyro-TWA. The HCW has both azimuthal and axial periodicities. It is able to generate an ideal dispersion curve that achieves a nearly constant group velocity at small wavenumber values and across a large frequency bandwidth by mode coupling. A mm-wave gyro-TWA using three-fold HCW has been developed at Strathclyde University [1]. It not only has the advantage of wide bandwidth but also to half the magnetic field strength at the interaction region as the beam-wave interaction occurs at the $2^{\text {nd }}$ cyclotron harmonic.

The gyro-TWA contained key components including cusp electron gun [2, 3], pillbox window [4], input coupler [5], elliptical polarizer, input and output windows [6, 7], HCIR, and solenoid systems. The axis-encircling electron beam in the gyro-TWA was generated by a cusp electron gun with a magnetic field reversal immediately in the front of the cathode. Its beam velocity ratio, $\alpha$ (ratio of transverse velocity to axial velocity, $v_{t} / v_{z}$ ), could be tuned by changing the magnetic field at the cathode surface through varying the driving currents of the solenoids. All the mm-wave components were optimized through numerical simulations and verified by measurement by using a vector network analyzer before and after they were assembled or vacuum brazed together.

In previous gyro-TWA experiments, the gain curve was measured at discrete frequency points. In this paper, an upgrade of the input signal generation was carried out, and the amplification of a frequency-swept signal was presented.

\section{EXPERIMENTAL SETUP}

A photo of the upgrade of the frequency-modulated input mm-wave signal is shown in Fig. 1 . An arbitrary waveform generator (model Keysight M8190A (1)) was used to generate signals with a maximum analog bandwidth of $5 \mathrm{GHz}$. The signal was then mixed with a $\sim 15.5 \mathrm{GHz}$ signal from a vector signal generator (model Agilent E8267D (2)). Such resultant signal was then used to drive a Quinstar solid-state amplifier (3) to generate a microwave signal in the frequency range of 90 to $96 \mathrm{GHz}$. The signal was attenuated by an Anritsu rotary vane attenuator (4) and measured by a waveguide harmonic mixer (5). The waveform can be processed by an oscilloscope (6) or a signal analyzer (7).

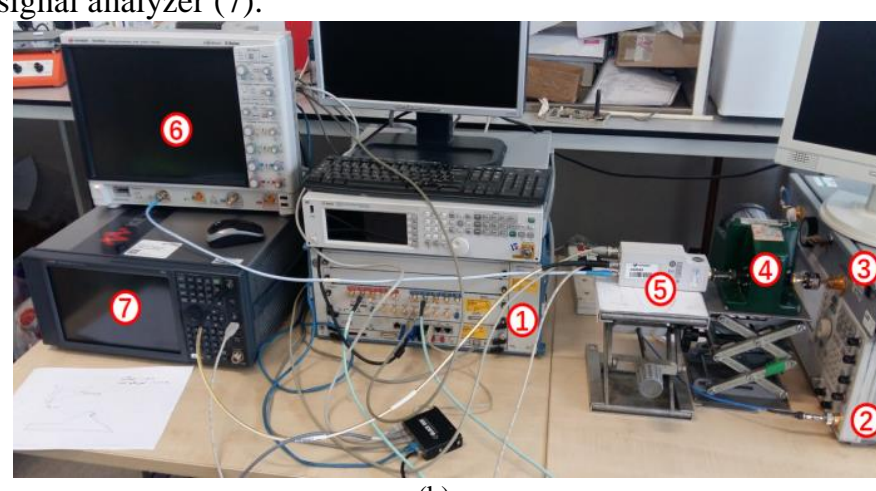

(b)

Fig. 1 A photo showing the setup for generation of the input mm-wave signal.

The instruments shown in Fig. 1 are dedicated and expensive. However, in the experiment, the pulsed voltage used to accelerate the electron beam was generated through the voltage breakdown (closing) of the spark-gap switch the electrical noise caused by the opening and closing of the switching was relatively large. The noise could be coupled into the low-power mm-wave devices through the capacitive coupling and the earth line. Extra attention was paid to the noise when carrying out the gyro-TWA experiment. The signal analyzer and oscilloscope were powered by an insulated transformer with an extra lowpass filter for isolation in the earth path. To avoid possible damage to the dedicated devices, all the signal instruments were shielded within a metal screened box. 
In Reference [1], the electron beam of $55 \mathrm{keV}, 1.5 \mathrm{~A}$ with an alpha of $\sim 1.0$ was used to achieve a gain of $\sim 37 \mathrm{~dB}$ with a bandwidth of at least $5.5 \mathrm{GHz}$. To demonstrate the versatility in operating the gyro-TWA the operating beam voltage was lowered to $40 \mathrm{kV}$. The $\alpha$ value of the electron beam was also reduced to $\sim 0.85$ in order to match the dispersion of the operating eigenwave. The required magnetic field was accordingly adjusted to maintain the resonance between the electron beam mode and the eigenwave. For comparison, the dispersion of the coupling $\mathrm{TE}_{21}$ and $\mathrm{TE}_{11}$ modes, resultant operating eigenwave and the electron beams in the two experiments are shown in Fig. 2. The particle-in-cell (PIC) simulations with the new parameter sets were performed using 3D PIC code MAGIC. The simulation results are shown in Fig. 4 , a gain of about $31 \mathrm{~dB}$ was predicted in the frequency range of 93 - $94 \mathrm{GHz}$ assuming an alpha spread of $10 \%$ and beam thickness of $0.1 \mathrm{~mm}$. The simulated gain for the two sets of the parameters that were used in these experiments is shown in Fig. 3 for comparison.

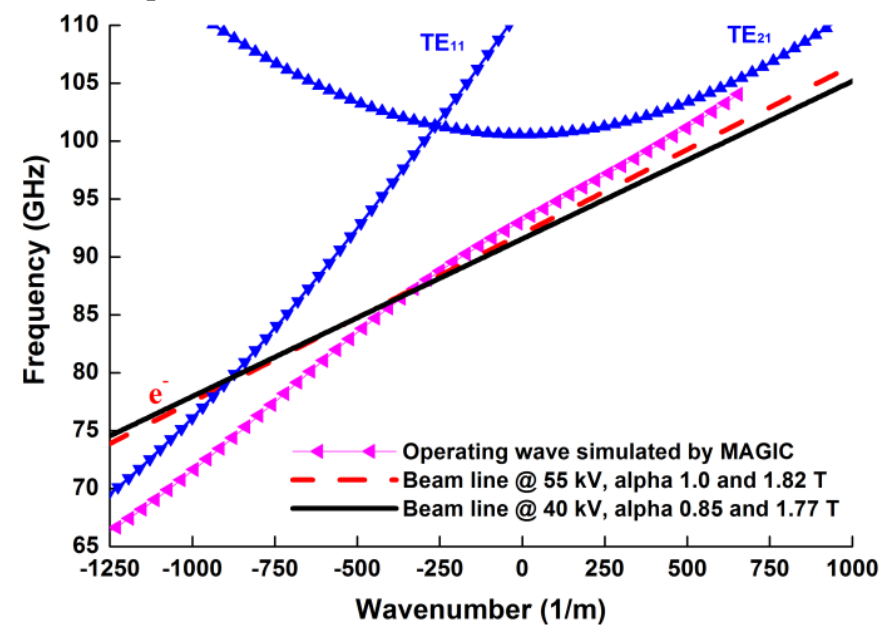

Fig. 2 Dispersion of the operating eigenwave and the electron beams in two separate experiments

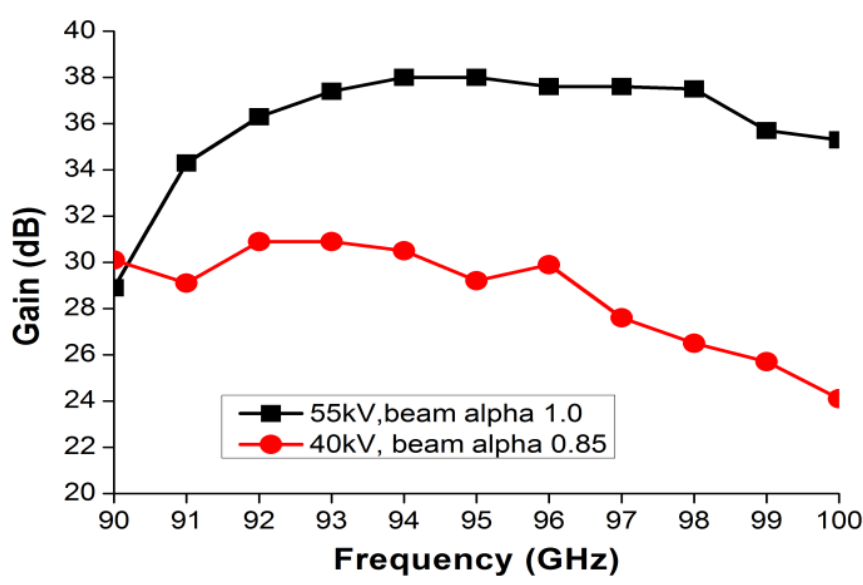

Fig. 3 Simulated gains from the two sets of beam parameters used in the separate experiments.

\section{MEASUREMENT RESULTS}

Due to the nonlinear effect from the six-time frequency multipliers in the solid-state source, it was found that it is difficult to achieve a desired signal beyond the bandwidth of 1 GHz. Therefore the frequency-swept signal was set to a bandwidth of $1.0 \mathrm{GHz}$ at the central frequency of $93.5 \mathrm{GHz}$. From Fig. 4(a), the noise of the frequency-swept signal generated from the solid-state source was relatively large due to the nonlinear behavior of frequency multipliers. The amplified signal in Fig. 4(a) showed clear frequency sweeping that aligned well with the input signal. The correlated mm-wave signal and the beam voltage is shown in Fig. 4(b). The spectrum of the amplified mm-wave signal in Fig. 4(c) showed a bandwidth close but smaller than $1 \mathrm{GHz}$. The phase of the input and amplified signal was processed and shown in Fig. 5. The phase information from the amplified signal of the gyro-TWA had the same trend as the input mm-wave signal. The noise was mainly from the variation of the pulsed beam voltage [8].

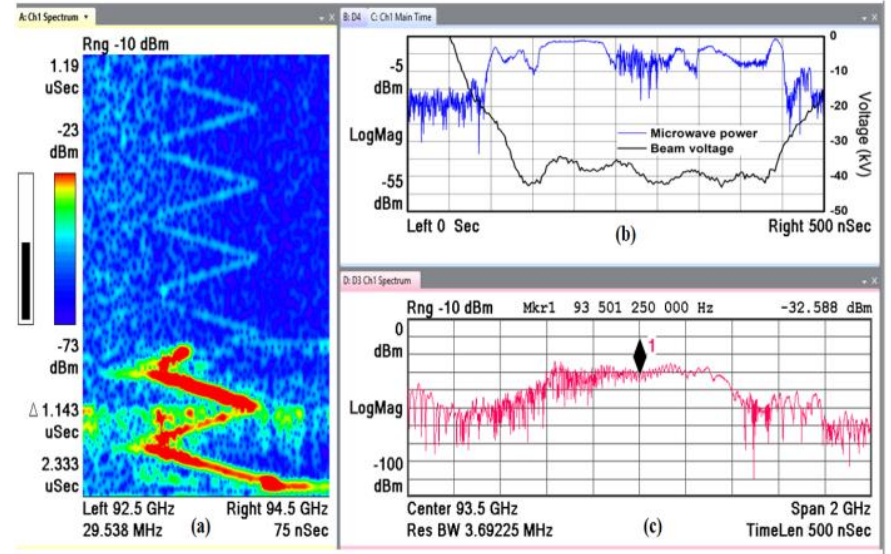

Fig. 4 Gyro-TWA experimental results with frequency-swept signal. (a) spectrogram, (b) correlation plot of the mm-wave signal and the acceleration voltage, and (c) spectrum of the mm-wave signal.

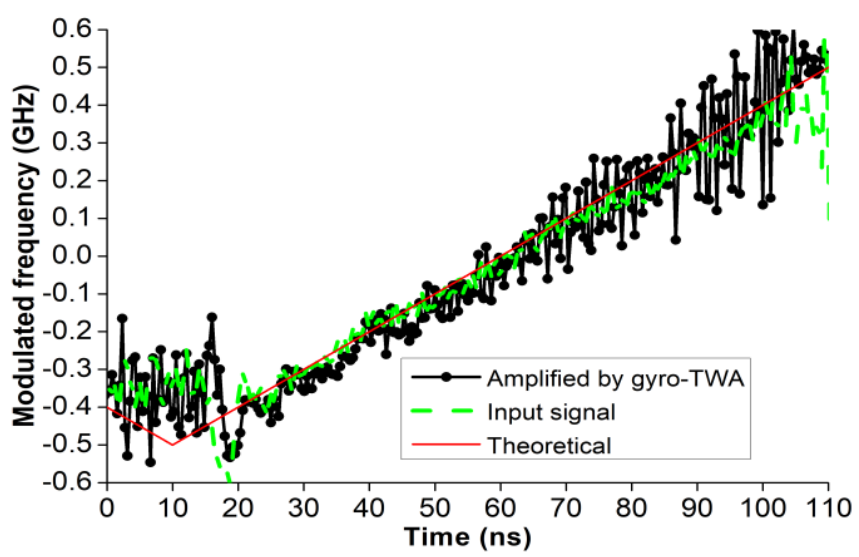

Fig. 5 Frequency sweeps of the input and output signal of the gyro-TWA experiment.

\section{CONCLUSION}

In this paper, a proof-of-principle experiment in the $\mathrm{W}$-band gyro-TWA with a frequency swept input signal has been carried out. A gain over $30 \mathrm{~dB}$ was achieved over the frequency band 
of $93.5-94.5 \mathrm{GHz}$. Frequency-swept signal with a bandwidth of $1 \mathrm{GHz}$ was generated as the input signal and the signal and successfully amplified with a gain of over $30 \mathrm{~dB}$. The measurement shows a good phase response of the W-band gyroTWA. The future improvements of the gyro-TWA include sourcing a wider bandwidth solid-state source as well as a more stable high-voltage power supply to drive the electron beam.

\section{ACKNOWLEDGMENT}

This work was supported by EPSRC UK (research Grant No. EP/G036659/1, EP/K029746/1) and STFC UK (research Grant No. ST/N002326/1 and ST/P001890/1).

\section{REFERENCES}

[1] W. He, C. R. Donaldson, L. Zhang, K. Ronald, A. D. R. Phelps, and A. W. Cross, "Broadband Amplification of Low-Terahertz Signals Using AxisEncircling Electrons in a Helically Corrugated Interaction Region," Phys. Rev. Lett., vol. 119, no. 18, p. 184801, Oct. 2017. DOI: 10.1103/PhysRevLett.119.184801

[2] C. R. Donaldson, W. He, A. W. Cross, F. Li, A. D. R. Phelps, L. Zhang, K. Ronald, C. W. Robertson, C. G. Whyte, and A. R. Young, "A cusp electron gun for millimeter wave gyrodevices," Appl. Phys. Lett., vol. 96, no. 14, p. 141501, 2010. DOI: 10.1063/1.3374888

[3] L. Zhang, W. He, C. R. Donaldson, and A. W. Cross, "Investigation on the optimal magnetic field of a cusp electron gun for a W-band gyro-TWA," Physics of Plasmas, vol. 25, no. 5, p. 053104, 2018/05/01 2018. DOI: $10.1063 / 1.5027070$

[4] L. Zhang, C. R. Donaldson, A. W. Cross, and W. He, "A pillbox window with impedance matching sections for a W-band gyro-TWA," IEEE Electron Device Letters, $\quad$ pp. $1-1, \quad 2018 . \quad$ DOI: 10.1109/LED.2018.2834859

[5] L. Zhang, W. He, C. R. Donaldson, J. R. Garner, P. McElhinney, and A. W. Cross, "Design and Measurement of a Broadband Sidewall Coupler for a W-Band Gyro-TWA," IEEE Trans. Microwave Theory Techn., vol. 63, no. 10, pp. 3183-3190, Oct. 2015. DOI: 10.1109/TMTT.2015.2464302

[6] L. Zhang, W. He, C. R. Donaldson, G. M. Smith, D. A. Robertson, R. I. Hunter, and A. W. Cross, "Optimization and Measurement of a Smoothly Profiled Horn for a W-Band Gyro-TWA," IEEE Trans. Electron Devices, vol. 64, no. 6, pp. 2665-2669, June 2017. DOI: $10.1109 /$ TED.2017.2687949

[7] C. R. Donaldson, P. McElhinney, L. Zhang, and W. He, "Wide-Band HE11 Mode Terahertz Wave Windows for Gyro-Amplifiers," IEEE Transactions on Terahertz Science and Technology, vol. 6, no. 1, pp. 108-112, June $2016 . \quad$ DOI: 10.1109/TTHZ.2015.2495221

[8] L. Zhang, C. R. Donaldson, P. Cain, A. W. Cross, and W. He, "Amplification of frequency-swept signals in a W-band gyrotron travelling wave amplifier," IEEE Electron Device Letters, pp. 1-1, 2018. DOI: 10.1109/LED.2018.2836868 